\title{
Edge Detection in Range Images of Piled Box-like Objects
}

\author{
Dimitrios Katsoulas and Andreas Werber \\ Albert-Ludwigs-University of Freiburg \\ Institute for Pattern Recognition and Image Processing \\ 79110 Freiburg, Germany \\ \{dkats, awerber\}@informatik.uni-freiburg.de
}

\begin{abstract}
We present a framework for edge detection in range images acquired by a time of flight laser sensor. Our edge detection approach is inspired by [7], in the context of which edge detection via scan line approximation with geometric parametric models is performed. The main drawback of this edge detector, namely the scan line over-segmentation problem is addressed by the introduction of a simple merging step. In addition, we incorporate a method for detection of the noisy data points created by the effect of laser beam splitting between surfaces of different ranges. Finally, a procedure for fine localization of the edge points is introduced. Experimental results on a variety of target object configurations demonstrate that our edge detection framework exhibits increased robustness and accuracy with regard to [7]. These characteristics in combination with the computational efficiency of our approach, allows for its usage as a component of a real time system for automatic unloading of piled box-like objects.
\end{abstract}

\section{Introduction}

Automatic unloading of piled box-like objects like those of fig. 3 (a-1),(b-1),(c-1), is undoubtedly of great importance to the industry. Existing systems e.g. [10],[15], employ primarily intensity imagery for dealing with the problem. Their main drawback is that they assume that the target objects are neatly placed. In addition, they are very sensitive to lighting conditions at the installation sites. Our approach towards the construction of a fully automatic object unloading system, involves usage of range imagery to deal with the problem [9]. Our system comprises, from the hardware point of view, an industrial robotic arm, on which a SICK LMS-200 [14] laser sensor is mounted. A range image of the object configuration is acquired by linearly moving the robotic arm above the object configuration, with the depth axis of the laser sensor pointing towards the objects.
Given the input range image, our system localizes the objects in the pile and the objects are grasped by the robot.

As discussed in [9], [8] accurate detection of the boundaries of the target objects in the pile, is of great importance for the robust performance of our system. In this paper, we propose an approach for edge detection in range images of our target object configurations. Our approach is inspired from the scan line approximation technique of [7]. This technique has been refined by the addition of a merging step as well as a method for detecting and discarding noisy range points, generated by laser beam splitting. The resulting framework exhibits advantages like computational efficiency accuracy and robustness.

In the section that follows our approach is discussed in detail. Section 2.1 gives an overview of the scan line approximation method and highlights its advantages and problems. Section 2.2 introduces our improved version for scan line approximation. Finally, in section 3, experimental results demonstrate the credibility of our approach.

\section{Boundary Detection via Scan Line Approx- imation}

A multitude of algorithms have been proposed for detecting edges in intensity images, based on local linear filtering ([5] p.175 - 181). The features of interest of these strategies however are the jump edges, since only this type of edges is encountered in intensity images. The situation is different in range images, since localization of jump and crease edges, as well as their combinations is required. The user is referred to [5] p. 473, for a definition of the different kinds of edges and their properties.

The majority of algorithms proposed to deal with edge detection in range images, (e.g. [2], [3]), define the sought edges in a local manner, and thereby use local operations for detecting them. Their advantage is the computational efficiency. However, local determination of boundary points often leads to false alarms in the presence of noise in the image. Incorporating global shape information in the edge de- 


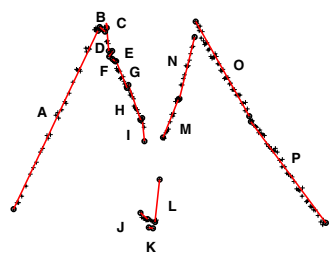

(a) Without merging

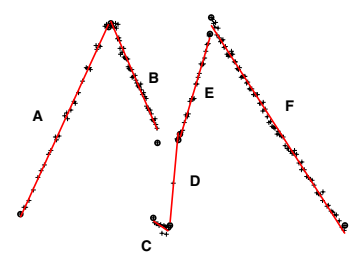

(b) Merging included

\section{Figure 1. Edge detection via scan line approx- imation with linear models}

tection process, which is available when knowledge about the geometry of the objects appearing in the image exists, is expected to increase the robustness of an edge detection algorithm.

\subsection{The Scan Line Approximation Technique}

Perhaps the most popular approach able to incorporate information about the target objects' shape in the edge detection process, is the one reported in [7]. The method approximates the rows and columns of the image, namely the scan lines, with one dimensional curves. Assuming that the objects in the image can be well modelled by implicit quadratic surfaces, approximation of the scan lines via quadratic polynomials is performed. For explaining the framework's internals, we assume that the sensor coordinate system is aligned to the range image, so that if $x, y, z$ the coordinates of a range point, the depth value $z$ is stored at the row $x$ and column $y$ of the image.

The framework involves usage of the scan line splitting technique of [4]: If $s, e$, and $m$ the respective positions of the start, end and mid point in the scan line, the corresponding depth values in these positions $z_{s}, z_{e}$ and $z_{m}$ are used to define the parameters of the approximating quadratic model via interpolation. Subsequently, the point with the maximum distance from the approximating model is retrieved. Assume $\max$ its position in the scan line. If the distance of this point to the model is lower than a user-defined threshold $\epsilon$, the points between $s$ and $e$ are considered to be satisfactory approximated by the model. Else, if the number of points contained in each segment with bounding indices $s, \max$ and $\max +1, e$ is bigger than the user defined threshold $\nu$, the splitting process is recursively applied on the two segments.

Candidate edge points are the end points of neighboring segments produced by the splitting process. Consider the neighboring segments $s_{1}, s_{2}$. Assume $e$ the end index of $s_{1}$ and $s=e+1$ the start index of $s_{2}$. Assume as well that $z=f_{1}(x), z=f_{2}(x)$ the parabolas approximating $s_{1}$, and $s_{2}$ respectively. If $\bar{x}=\frac{s+e}{2}$, then the jump and crease edge strengths of candidate edge points at $e$ and $s$ are given by (1),(2) respectively. The candidate points whose strength is bigger than a user defined threshold are the output edge points.

$$
\begin{aligned}
\mathrm{JES} & =\left|f_{1}(\bar{x})-f_{2}(\bar{x})\right| \\
\mathrm{CES} & =\cos ^{-1} \frac{\left(-f_{1}^{\prime}(\bar{x}), 1\right)\left(-f_{2}^{\prime}(\bar{x}), 1\right)^{T}}{\left\|\left(-f_{1}^{\prime}(\bar{x}), 1\right)\right\|\left\|\left(-f_{2}^{\prime}(\bar{x}), 1\right)\right\|}
\end{aligned}
$$

The approach exhibits many advantages: Computational efficiency is the outcome of the fast scan line segmentation via splitting, and of the fact that interpolation is used for the approximation. Robustness and accuracy in the localization of the edge points, is the outcome of incorporating global shape information in the process. Classification of a range point as edge point, does not any more depend on local information but on the parameters of neighboring approximating models, the estimation of which is influenced by a big number of range points. Finally, the approach is simple and easy to implement. The thresholds used, namely the minimum number of points per segment $\nu$, the maximum allowable distance of a segment's point to the approximating model $\epsilon$, and the crease and jump edge strength thresholds, have a straightforward interpretation, which renders their tuning relatively easy.

A drawback of the method however is the scan line oversegmentation, illustrated in Fig. 1 (a), where, for reasons of simplicity, linear instead of parabolic models have been employed for the approximation. In this image, data points are represented by crosses, and the segment end points are shown in bold. Ideally, all segments inclusively between each of C-I, J-K, M-N and O-P should be replaced with one segment. In [7], the problem is implicitly addressed by the fact that not all segment end-points are delivered as output of the edge detector, but only those with large edge strengths. The end point of segment $\mathbf{O}$ for example is determined not to be a valid edge point, since the angle between $\mathbf{O}$ and $\mathbf{P}$ is almost $\pi$. However the problem cannot be entirely solved in this way: Boundaries of small noisy segments are falsely classified as edge points, since their crease edge strength value can be relatively large. This is the case for the boundaries of the segments $\mathbf{E}, \mathbf{F}$, for example. Experimentation with our sensor showed that such cases cause a significant degradation of the boundary detection output. Thereby, a solution to the over-segmentation is of significant importance to our application.

Another problem observed, has not entirely to do with the segmentation approach itself, but with the fact that time of flight laser sensors output noisy points in the areas of depth change, due to splitting of the laser beam [1]. Fig. 2 (a) illustrates: The noisy points are assigned to the segments $\mathbf{B}$ and $\mathbf{C}$ in the figure. Since these segments do not correspond to real objects, edge strength values calcu- 
lated at their boundaries are unreliable, and should not be included in the output of the edge detection process. Detecting such noisy segments and discarding the respective edge information, is expected to improve the performance of the edge detection algorithm.

We employ the scan line approximation technique for edge detection. However, there are differences from the original approach of [7]. A merging step is incorporated to deal with the over-segmentation problem. In addition, a simple technique for detecting segments with noisy points in the areas of depth change is devised. Finally, a method for more accurate localization of the edge points in the image is introduced.

\subsection{Our Approach for Edge Detection}

A possible strategy for dealing with scan line oversegmentation is to increase the approximation threshold $\epsilon$. Unfortunately, this may lead to the inverse effect, namely scan line under-segmentation, which is more difficult to handle. A solution to the problem is described in [12]. In this work, a method for scan line segmentation based as well on approximation with geometric models is presented. The main difference with [7], is that the segmentation is attempted without the usage of the threshold $\epsilon$, so that segmentation becomes almost non-parametric. Scan line splitting continues until the next splitting step creates a segment with less than $\nu$ points. The resulting over-segmentation is handled by the introduction of a very simple merging step: For each segment of the final segmentation, the point with the maximum distance $d$ from the approximating model is considered. A significance value $\mathbf{S}$ is then assigned to the segment, defined as the ratio between the length of the segment $L$ divided by $d$, as in (3).

$$
S=\frac{L}{d}
$$

According to the merging procedure each segment is combined sequentially with the previous, the next and both the previous and next segments and for each combination the value $\mathbf{S}$ is computed. If one of the combinations results in a bigger $\mathbf{S}$ than the one of the candidate segment, the corresponding segments are merged. Note that definition of the significance measure as in (3), implies $\nu=P+1$, where $P$ the number of parameters of the approximating model,so that infinite significance values are avoided.

The advantage of the merging approach is that its realization does not require introduction of additional thresholds. Experimentation with the method showed that it produces superior results when long, well approximated segment outnumber the small, probably noisy segments. In [12], this is not the case: the number of small segments is relatively large, because splitting is performed exhaustively. The splitting threshold $\epsilon$ guarantees the existence of longer segments

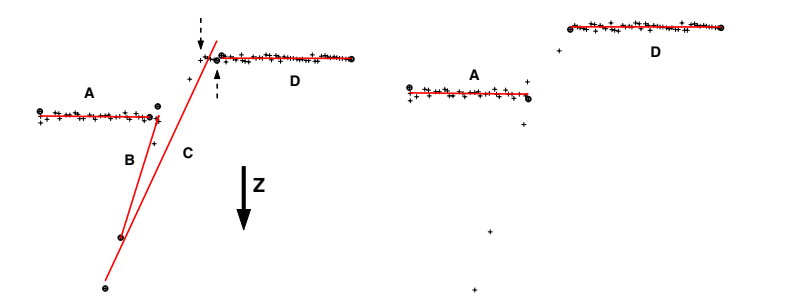

$\begin{array}{ll}\text { (a) Approximation output } & \text { (b) Final result }\end{array}$

Figure 2. Detection of noisy segments

in our case. Application of the merging step in the scan line of fig. 1 (a), is depicted in fig. 1 (b). Note that fitting is used, instead of interpolation for model approximation, in order to generate the segmentation of the figure. Using fitting results into more accurately approximated segments, and thus in more reliable significance values. Thereby, usage of fitting improves the effectiveness of the merging process.

We employ a simple but effective heuristic approach to detect and discard segments corresponding to spurious data points caused by the effect of laser beam splitting between surfaces of different ranges. These points are aligned and can be accurately modeled by linear models, which are almost parallel to the range axis $\mathbf{Z}$ of the sensor coordinate system. This is illustrated in Fig. 2 (a). The noisy segments $\mathbf{B}, \mathbf{C}$ in the figure, can be easily identified: The angle formed between each segment and the depth axis $\mathbf{Z}$ of the sensor coordinate system is computed. If the angle is smaller than a predefined threshold, the segment is considered noisy and discarded. Application of this filtering to the data of fig. 2 (a), is depicted in fig. 2 (b). Note in the figure, that the meaningless crease edges generated between the segments A-B and C-D in fig. 2 (a) are replaced by a jump edge expressing the height difference between the segments A-D, as desired. We have experimented with more sophisticated techniques for discarding these noisy points (e.g. [1]), which did not prove to deliver better results for our data sets. In addition, combining edge detection and noise filtering in one process saves computational costs.

Note, that in fig. 2 (a), some points expected to belong to segment $\mathbf{D}$ (namely the points between the dashed arrows in the figure), are assigned to the noisy segment $\mathbf{C}$ by the splitting process. The reason for this is that the splitting process is not allowed to generate segments with less than $\nu$ points. This problem of minor point misclassification during splitting is observed in [7], and a heuristic solution is proposed for candidate crease edge points only. For improving the position of candidate jump edge points after noisy segment deletion, points from the deleted noisy segment $\mathbf{C}$ which have a distance lower than $\epsilon$ from the approximating model of the neighboring segment $\mathbf{D}$ are added to the segment $\mathbf{D}$. The new starting point of $\mathbf{D}$ is marked 
as candidate edge point. As seen in fig. 2 (b), the candidate edge point is accurately recovered in this way.

Our overall approach for edge detection acts on all the rows and columns (scan lines) of the input range image. Linear models are employed for modeling our almost planar target objects, and model fitting is utilized for the approximation. For every scan line, splitting is performed. A merging step follows. Subsequently, noisy segments are identified and discarded. The boundary points of segments neighboring noisy segments are accurately localized. Finally, edge strength values are calculated and the points with high values are returned as edge points. In the paragraph the follow, experimental results give the reader an overview of the performance of our approach.

\section{Experimental results}

We conducted experiments with about 20 range images corresponding to box-like object configurations. In fig. 3 representative results are illustrated. The first row of the figure contains the intensity images of three object configurations. In the second row, edge detection results obtained via application of [7] to the corresponding range images are depicted. The detected edges are superimposed to the range images. The third row of the figure depicts the results of our approach. For all experiments $\epsilon=15$ and $\nu=3$ was used. By inspecting the figure, one can get a clear impression of the improvement, concerning the edge localization accuracy, introduced by our approach. Note as well that the localization accuracy does not deteriorate sharply when dealing with non rigid objects likes those of fig. 3 (b-1),(c-1), which demonstrates the robustness of our approach.

$$
F O M=\frac{M}{N} \times \sum_{i=0}^{N} d_{i}^{2}
$$

In order to acquire a more clear overview of the performance of our approach, we performed a quantitative comparison of our strategy to [7]. Assuming $N$ the number of points in a scan line, $M$ the number of segments and $d_{i}$ the distance of the point at position $i$ from the model approximating the point, we used the quantity defined in (4) to perform the comparison. The quantity FOM has been introduced in [13] and used among others in [12] for comparing segmentation results. Lower values of FOM correspond to better segmentations.

The comparison results are illustrated in table 1 . The first and second columns of the table correspond to the average FOM per scan line obtained by [7] and our approach, respectively. The rows of the table correspond to the range images where the methods have been applied. The first row concerns the card board boxes configuration (fig. 3 (a$1)$ ), the second row the configuration of box like objects

\begin{tabular}{|l|c|c|}
\hline & Jiang/Bunke [7] & Our Approach \\
\hline Fig. 3 (a-1) & 1345 & 755 \\
\hline Fig. 3 (b-1) & 1774 & 1074 \\
\hline Fig. 3 (c-1) & 2375 & 1198 \\
\hline
\end{tabular}

Table 1. Comparison results

(fig. 3 (b-1)), and the third row the configurations of sacks (bags) of fig. 3 (c-1). Note, that as highlighted in [11], the $F O M$ measure favors approximations with larger number of segments. Since the number of segments produced by [7] is larger than those obtained by our approach, due to the fact that the latter includes a merging step, the FOM favors the result obtained by application of [7]. Despite this, for all cases the average $F O M$ per scan line obtained by our approach is lower.

Regarding computational efficiency, execution of our method took about 2 seconds in a Pentium $I V$ processor of $2.8 \mathrm{GHz}$, which implies that our technique is suitable for real time use.

\section{Conclusions}

We presented an approach for model based edge detection in range images of piled box-like objects, based on scan line approximation with linear models. Our strategy for edge detection exhibits computational efficiency accuracy and robustness. In the near future, we plan to continue experimentation with our approach by considering a bigger variety of target objects and configurations. In addition, we plan to compare our edge detection approach with other edge detectors, using ground truth segmented images of various sensors, in the context of the range image segmentation comparison framework of [6].

\section{References}

[1] M. Adams. Amplitude Modulated Optical Range Data Analysis in Mobile Robotics. In IEEE International Conference on Robotics and Automation: Volume 2, pages 8-13, May 1993.

[2] E. Al-Hujazi and A. Sood. Range Image Segmentation with Applications to Robot Bin-picking using Vacuum Gripper. IEEE Trans. on Systems Man and Cybernetics, Vol. 20, No. 6, 1990.

[3] P. Boulanger, F. Blais, and P. Cohen. Detection of Depth and Orientation Discontinuities in Range Images using Morphology. In 10th International Conference on Pattern Recognition, Vol. B, pages 729-732, 1990.

[4] R. Duda and P. Hart. Pattern Recognition and Scene Analysis. John Wiley and Sons, 1973.

[5] D. Forsythe and J. Ponce. Computer Vision: A Modern Approach. Prentice Hall, 2003. 


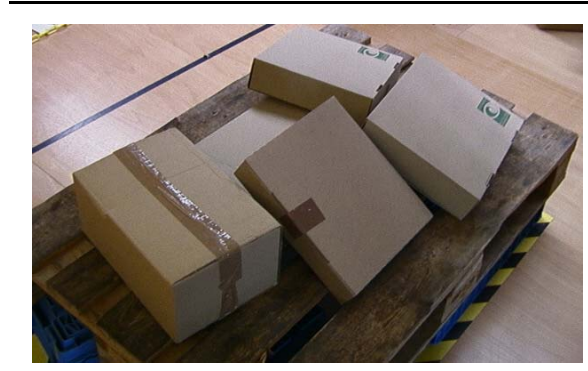

(a-1)

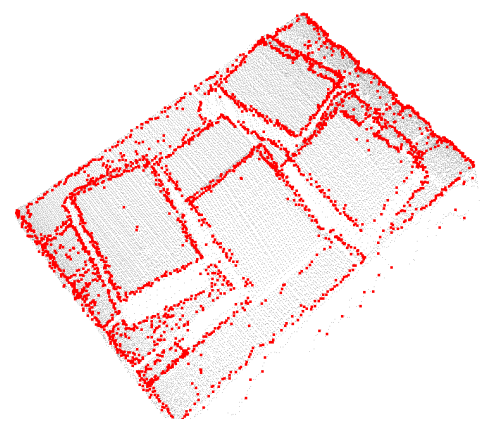

(a-2)

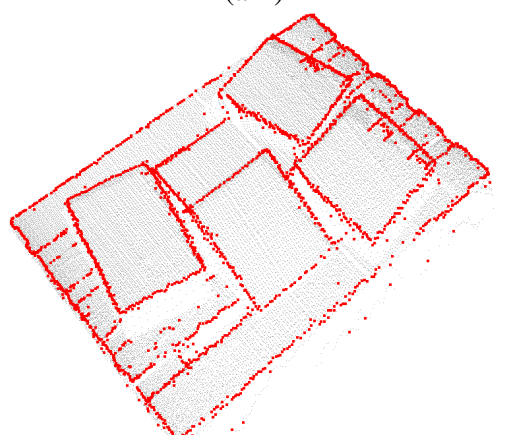

(a-3)

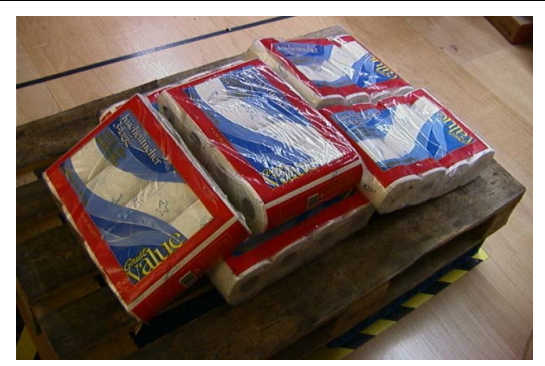

(b-1)

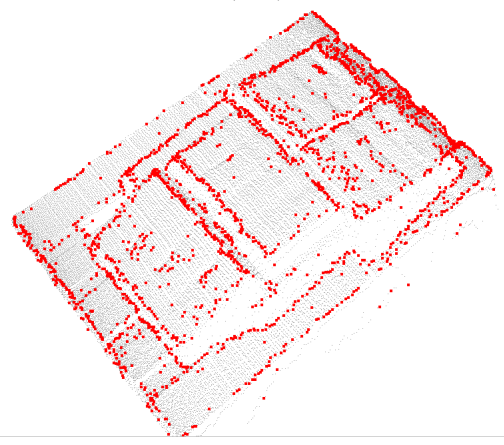

(b-2)

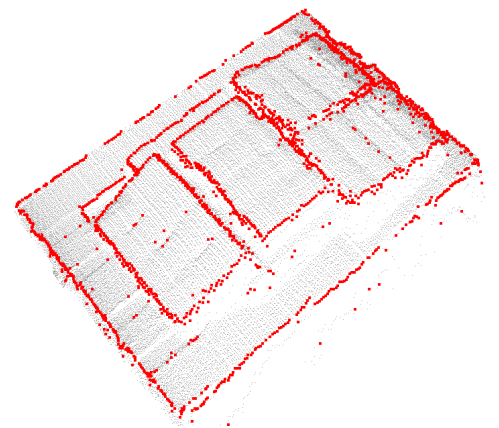

(b-3)

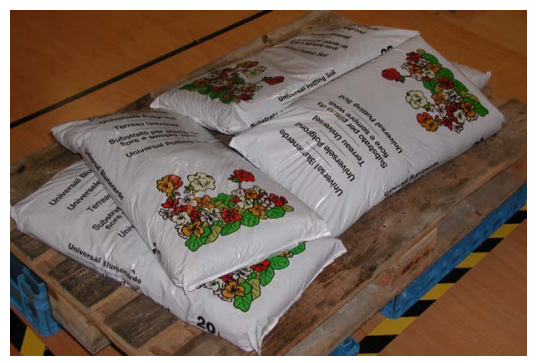

(c-1)

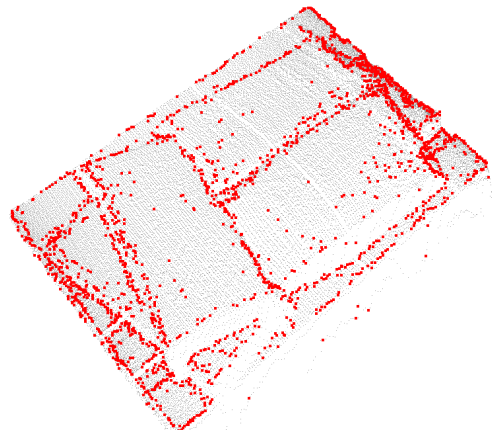

(c-2)

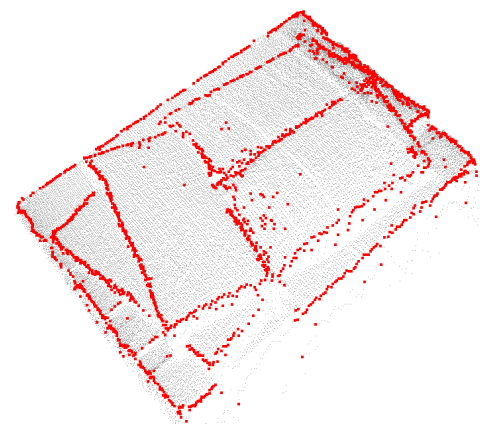

(c-3)

Figure 3. Edge Detection on Box-like object configurations

[6] A. Hoover. An Experimental Comparison of Range Image Segmentation Algorithms. IEEE Trans. PAMI, 18(7):637689, 1996.

[7] X. Jiang and H. Bunke. Edge Detection in Range Images Based on Scan Line Approximation. Computer Vision and Image Understanding: CVIU, 73(2):183-199, Feb. 1999.

[8] D. Katsoulas. Localization of Piled Boxes by Means of the Hough Transform. In Pattern Recognition, Proc. of 25th DAGM Symposium, pages 44-51, Magdeburg, Germany, Sept. 2003.

[9] D. Katsoulas. Reliable Recovery of Piled Box-like Objects via Parabolically Deformable Superquadrics. In IEEE International Conference on Computer Vision, pages 931-938, Nice, France, Oct. 2003.

[10] D. Newcorn. Robot Gains Eyesight. Packaging World, Oct. 1998.
[11] P. Rosin. Techniques for Assessing Polygonal Approximations of Curves . IEEE Trans. on Pattern Analysis and Machine Intelligence, Vol. 19, No. 6, 1997.

[12] P. Rosin and G. West. Nonparametric Segmentation of Curves into Various Representations. IEEE Trans. on Pattern Analysis and Machine Intelligence, Vol. 17, No. 12, 1995.

[13] D. Sarkar. A Simple Algorithm for Detection of Significant Vertices for Polygonal Approximation of Chain-Coded Curves. Pattern Recognition Letters, 14:959-964, 1993.

[14] SICK-GmbH. LMS200 technical description.

[15] S. Wienand. Robot Vision for Depalletizing of Mixed Pallet Loads. Machine Vision Online, Oct. 2003. 